Supplement of Weather Clim. Dynam., 1, 261-275, 2020

https://doi.org/10.5194/wcd-1-261-2020-supplement

Weather and

(c) Author(s) 2020. This work is distributed under the Creative Commons Attribution 4.0 License.

(c) (1)

Supplement of

\title{
Intermittency of Arctic-mid-latitude teleconnections: stratospheric pathway between autumn sea ice and the winter North Atlantic Oscillation
}

Peter Yu Feng Siew et al.

Correspondence to: Peter Yu Feng Siew (yu.siew@uib.no)

The copyright of individual parts of the supplement might differ from the CC BY 4.0 License. 


\section{List of Figures}

1 As in Fig. 3, but showing the beta coefficients (colours) of all detected casual linkages. Note that no simultaneous relationships are shown here, including $\uparrow \mathrm{URALS}_{D e c} \Rightarrow \uparrow \mathrm{V}^{*} \mathrm{~T}^{*}{ }_{\text {Dec }}$ (coloured horizontal line in Fig. 3a). . . . . . . . . . . . . . . . 2

2 The unaggregated version of the half-monthly CEN, equivalent to Fig. S1b after aggregation. . . . . . . . . . . . . . . . 3

3 Pentad version of the monthly (Fig. 3a, S1a) and half-monthly (Fig. 3b, S1b) CENs. A 1\% significance level is used in the second step (see Data and Methods 2.2). A maximum lag of 12 pentads (i.e., 2 months) is allowed. Autocorrelation is not used to reject causal linkages in the partial correlation tests here. . . . . . . .

4 As in Fig. 3, but using AIC with another significance set (5\%, $10 \%, 20 \%, 30 \%, 40 \%, 50 \%) \ldots \ldots \ldots \ldots$

5 The correlation between October (red) and November (blue) BarentsKara sea ice indices, and NAO from October to March. Outer grey lines show significant correlations at a $5 \%$ level using a two-

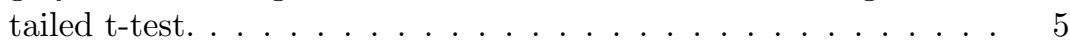

6 As in Fig. 3a, but highlighting all ICE-NAO stratospheric and tropospheric pathways. . . . . . . . . . . . 6

7 Same as Fig. S1a, but additionally showing the occurrences rates of all linkages in the bootstrapping test. . . . . . . . . . 7

8 Results of the pentad CEN analysis assessing relationships between downward longwave radiation (IR), Barents-Kara sea ice (ICE) and Urals sea level pressure (URALS). A maximum lag of 2 pentads is allowed. Autocorrelation is not calculated. Fig. 7 is aggregated from this figure by summing the number of times each linkage appears in individual months. . . . . . . . . . . .

9 Monthly CEN as in Fig. S1a, but with the addition of downward longwave radiation $($ IR) . . . . . . . . . . . . . . . 9 9 


\section{(a) Monthly CEN}

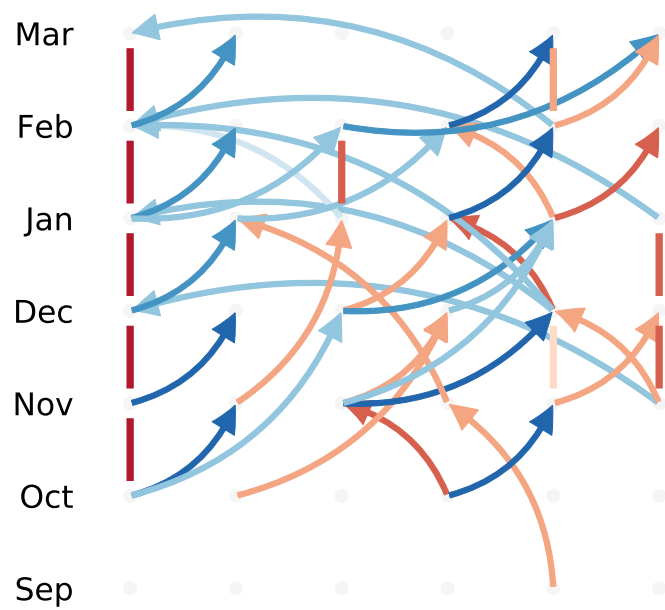

Sep (b) Half-monthly CEN

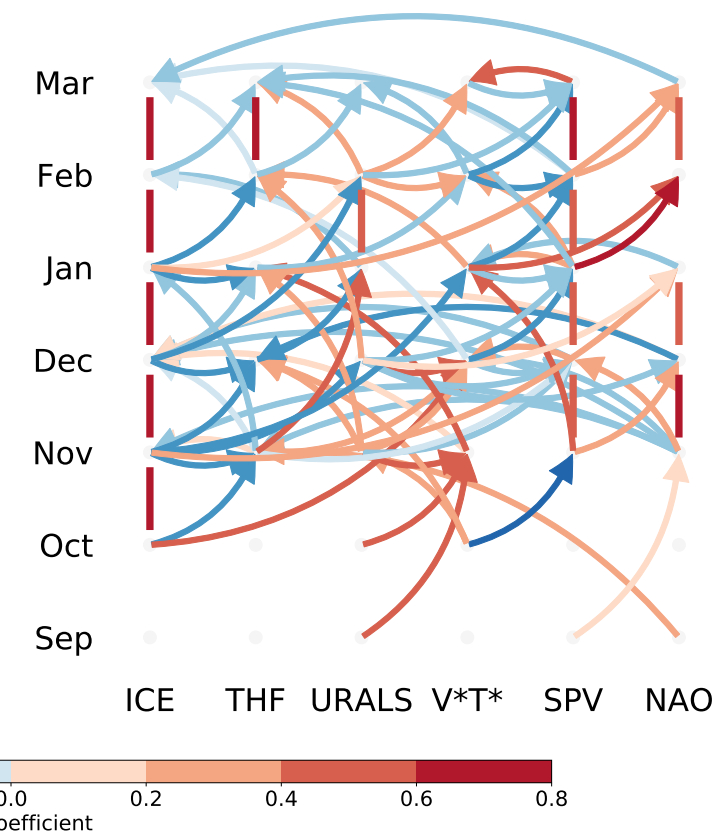

Figure S1: As in Fig. 3, but showing the beta coefficients (colours) of all detected casual linkages. Note that no simultaneous relationships are shown here, including $\uparrow \mathrm{URALS}_{D e c} \Rightarrow \uparrow \mathrm{V}^{*} \mathrm{~T}^{*}{ }_{\text {Dec }}$ (coloured horizontal line in Fig. 3a). 


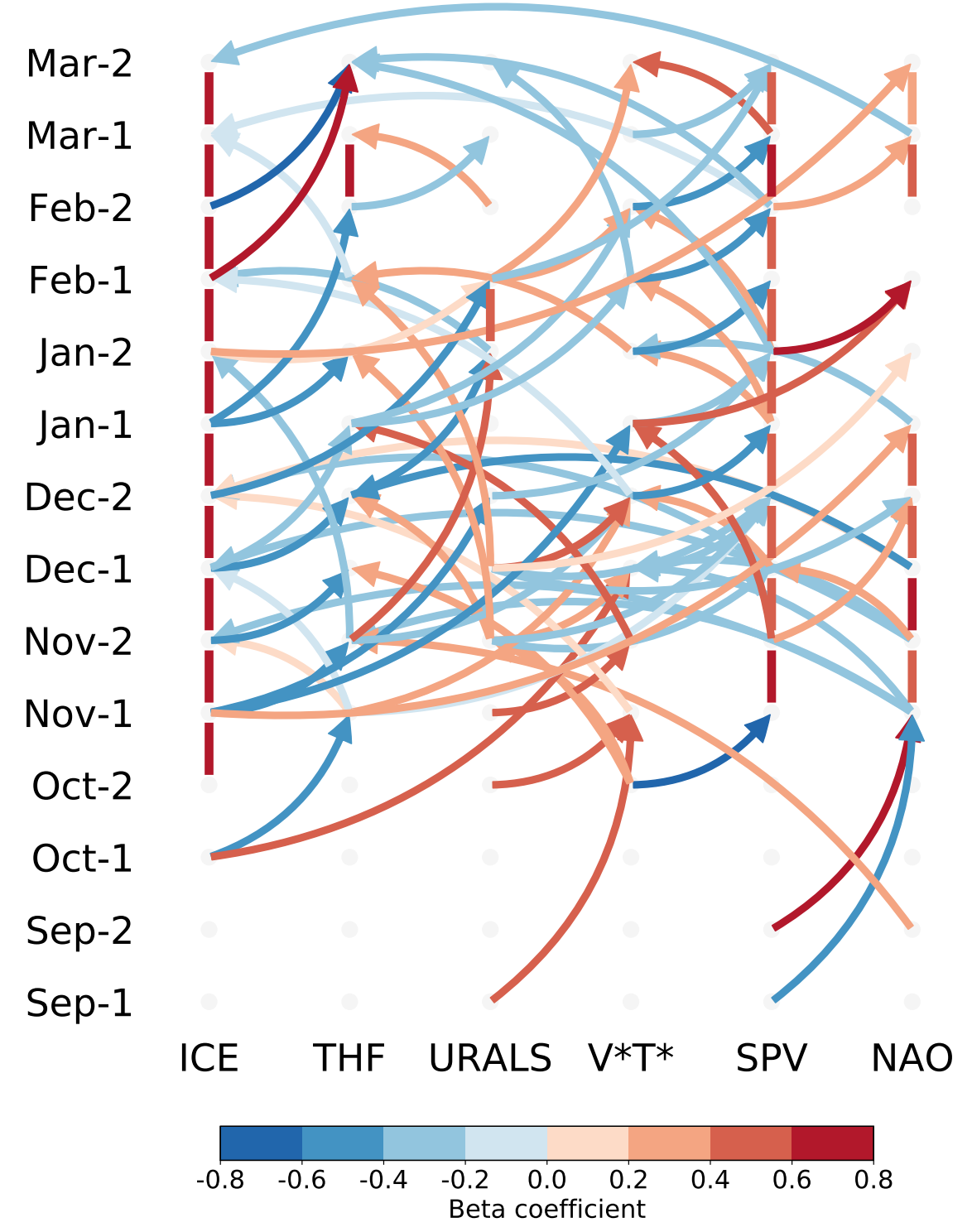

Figure S2: The unaggregated version of the half-monthly CEN, equivalent to Fig. S1b after aggregation. 


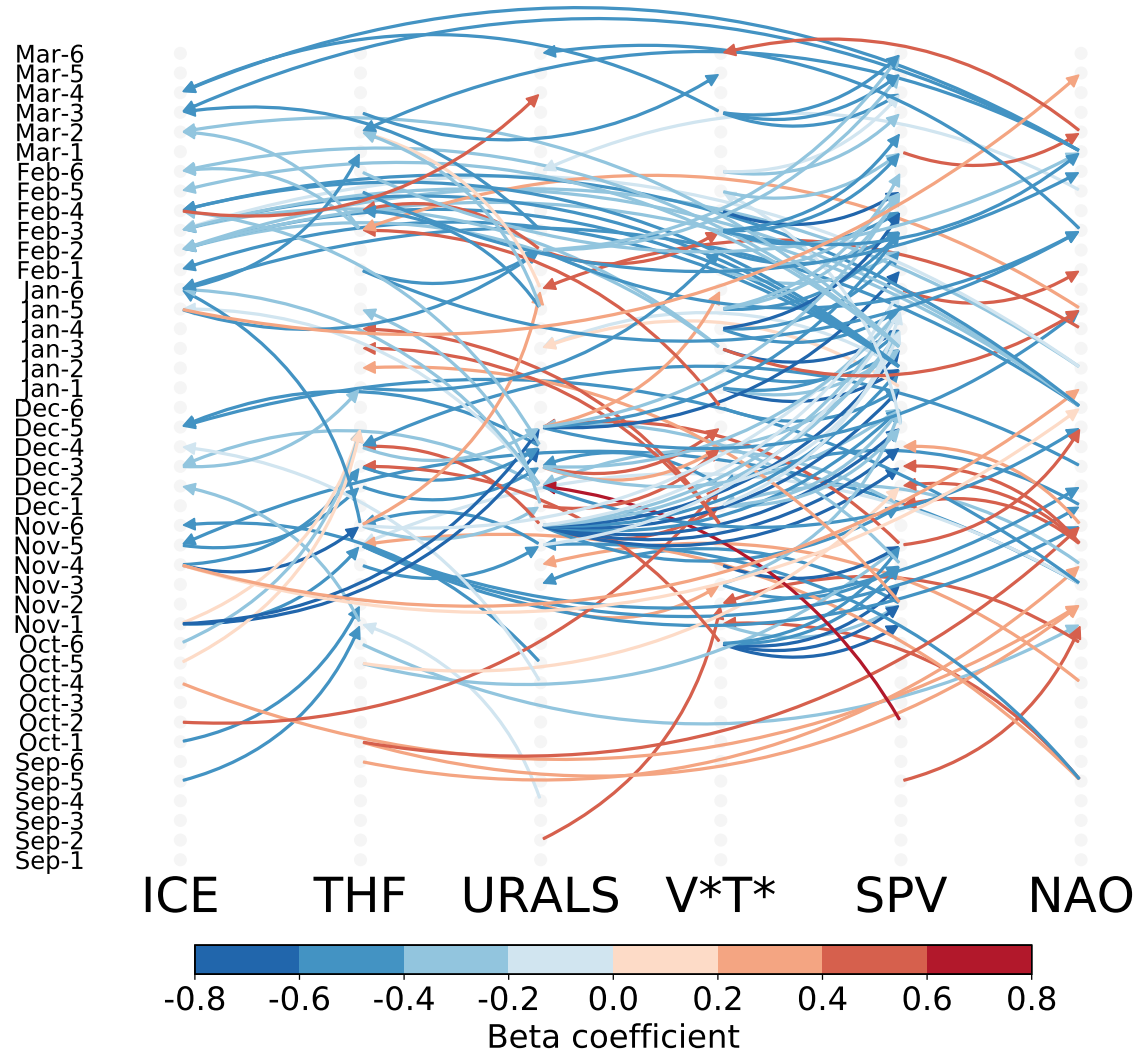

Figure S3: Pentad version of the monthly (Fig. 3a, S1a) and half-monthly (Fig. 3b, S1b) CENs. A 1\% significance level is used in the second step (see Data and Methods 2.2). A maximum lag of 12 pentads (i.e., 2 months) is allowed. Autocorrelation is not used to reject causal linkages in the partial correlation tests here. 
(a) Monthly CEN

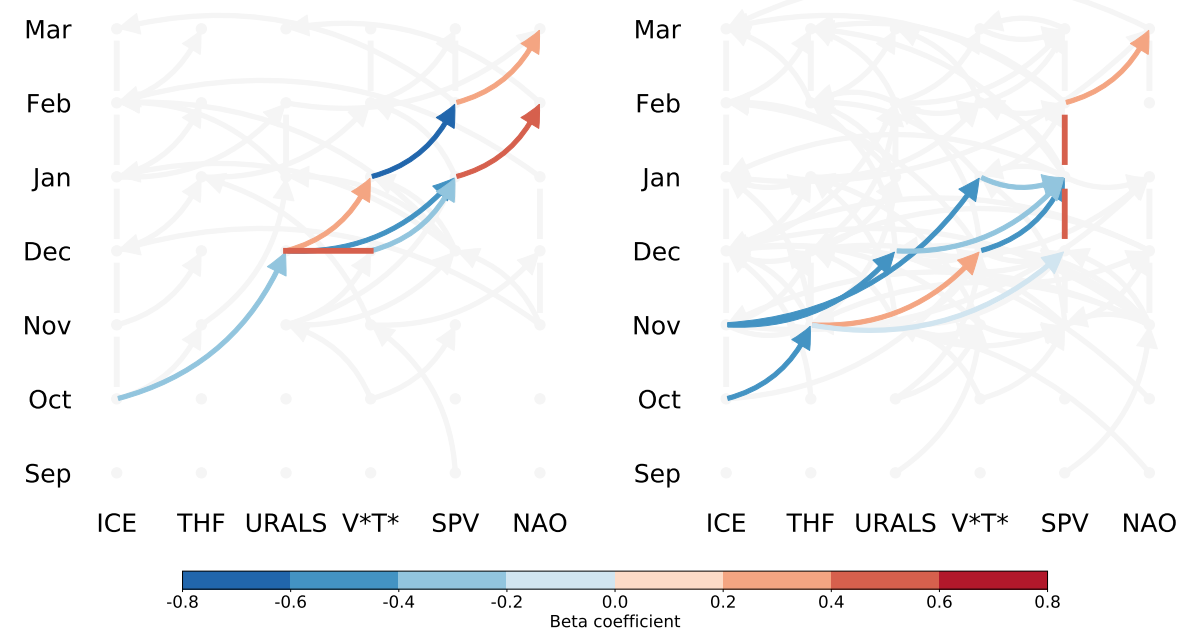

Figure S4: As in Fig. 3, but using AIC with another significance set $(5 \%, 10 \%$, $20 \%, 30 \%, 40 \%, 50 \%)$.

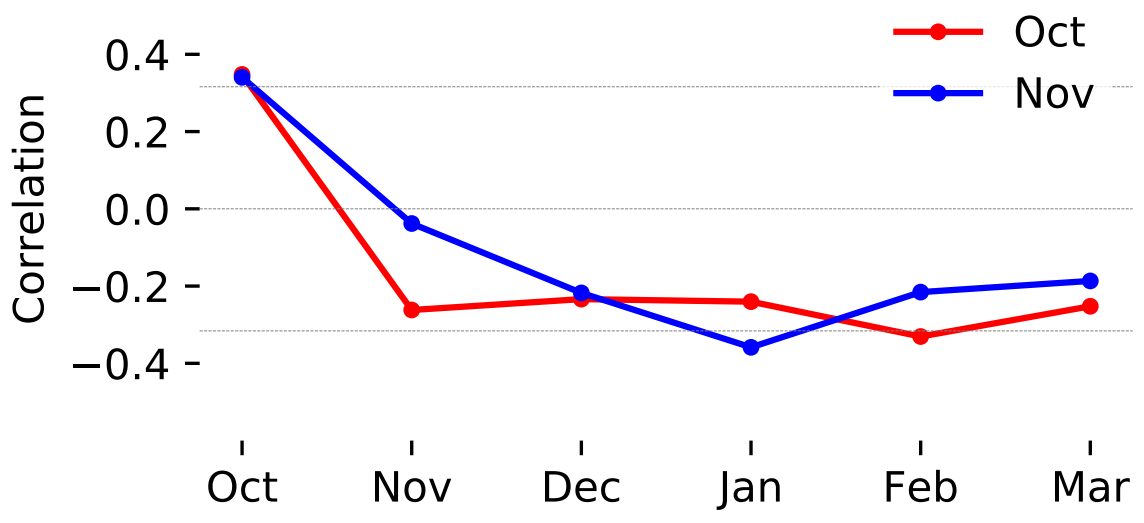

Figure S5: The correlation between October (red) and November (blue) BarentsKara sea ice indices, and NAO from October to March. Outer grey lines show significant correlations at a $5 \%$ level using a two-tailed t-test. 


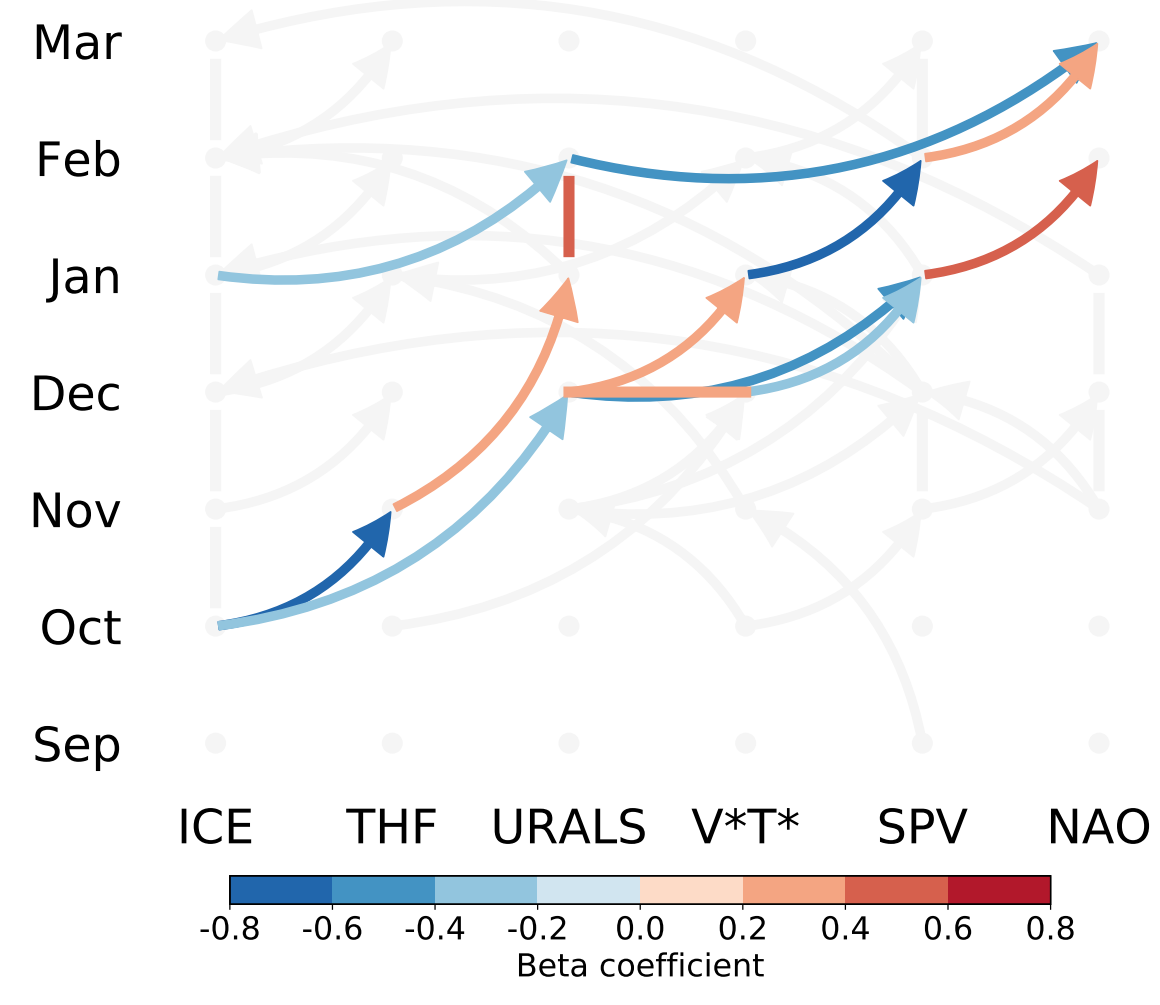

Figure S6: As in Fig. 3a, but highlighting all ICE-NAO strato-spheric and tropospheric pathways. 


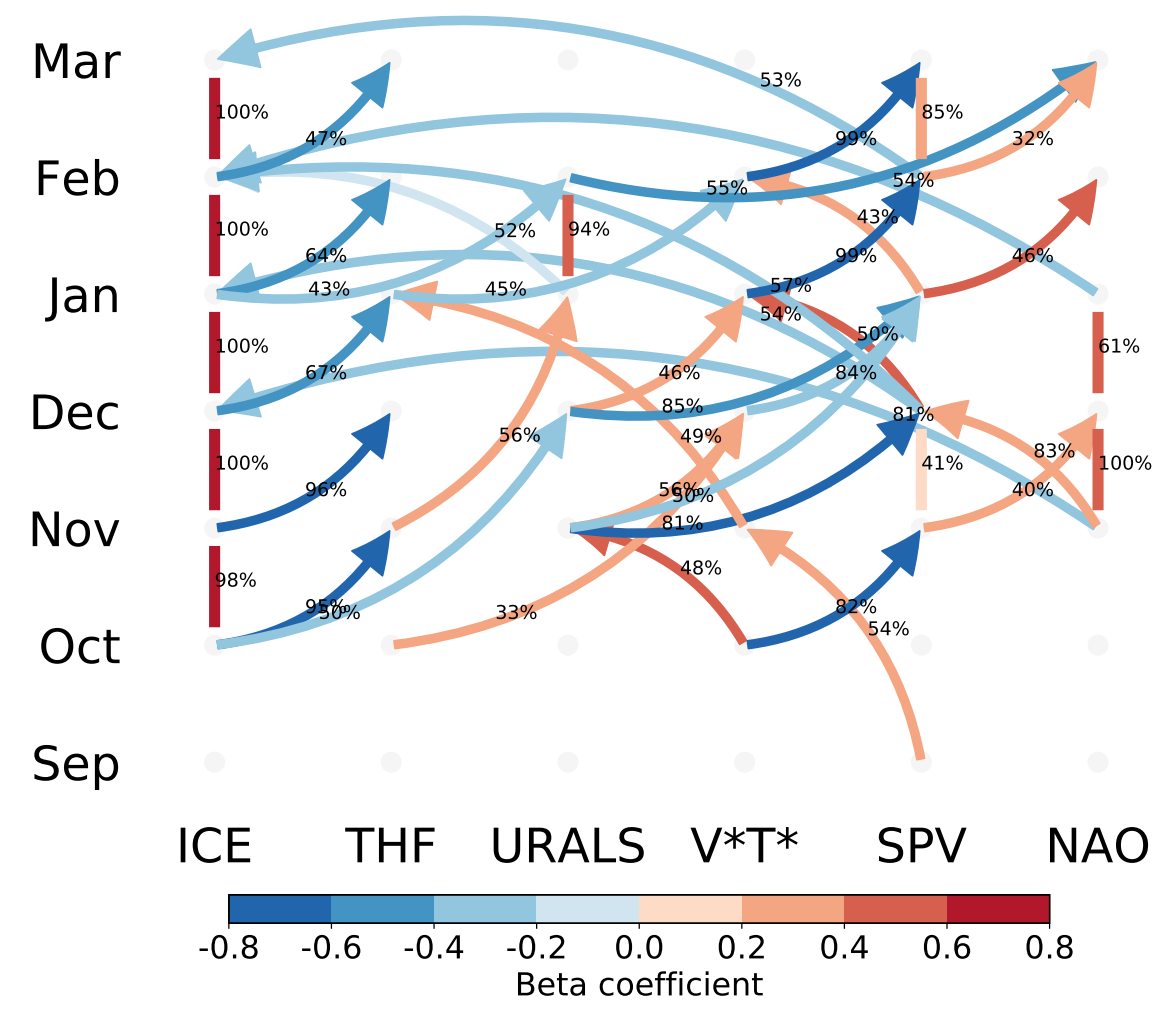

Figure S7: Same as Fig. S1a, but additionally showing the occur-rences rates of all linkages in the bootstrapping test. 

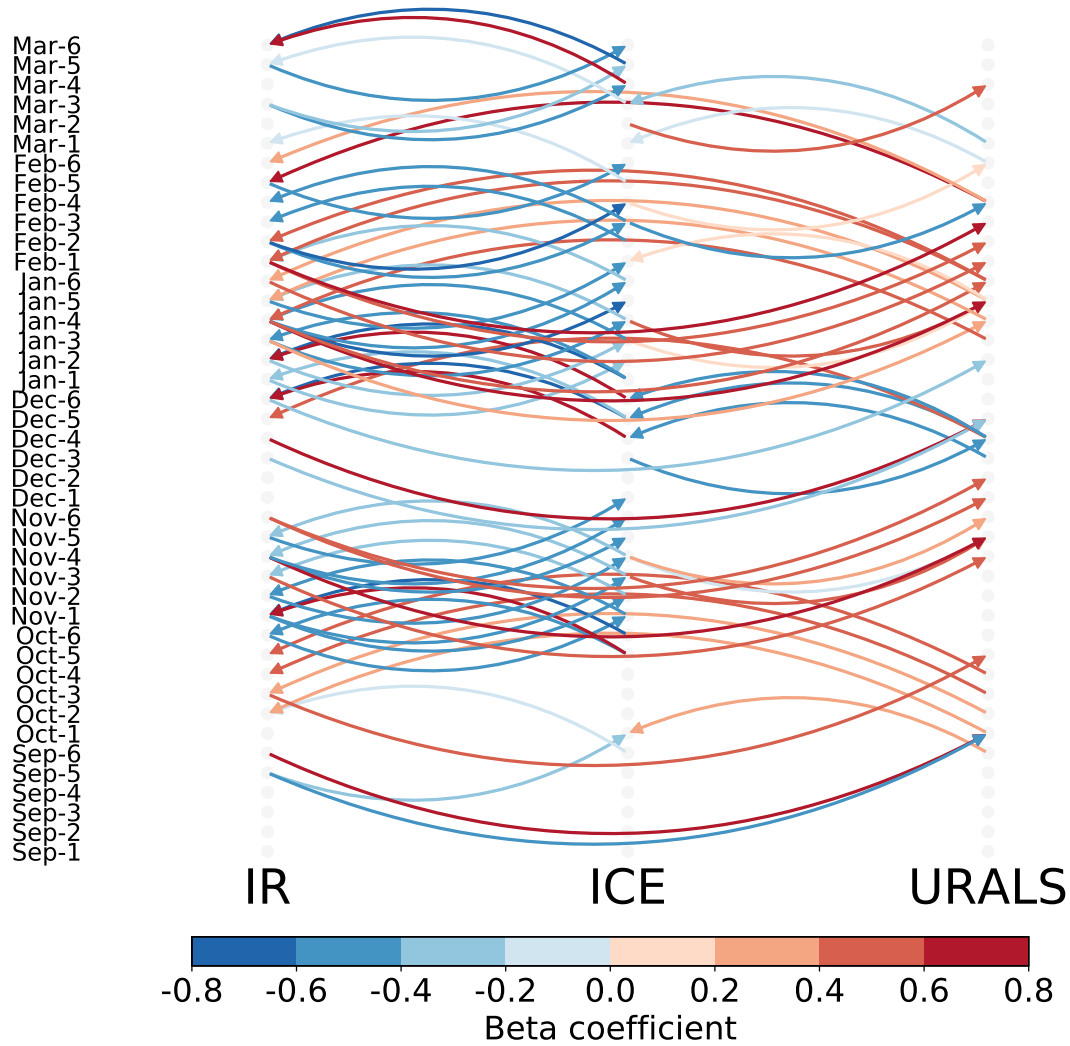

Figure S8: Results of the pentad CEN analysis assessing relation-ships between downward longwave radiation (IR), Barents-Kara sea ice (ICE) and Urals sea level pressure (URALS). A maximum lag of 2 pentads is allowed. Autocorrelation is not calculated. Fig. 7 is aggregated from this figure by sum-ming the number of times each linkage appears in individual months. 


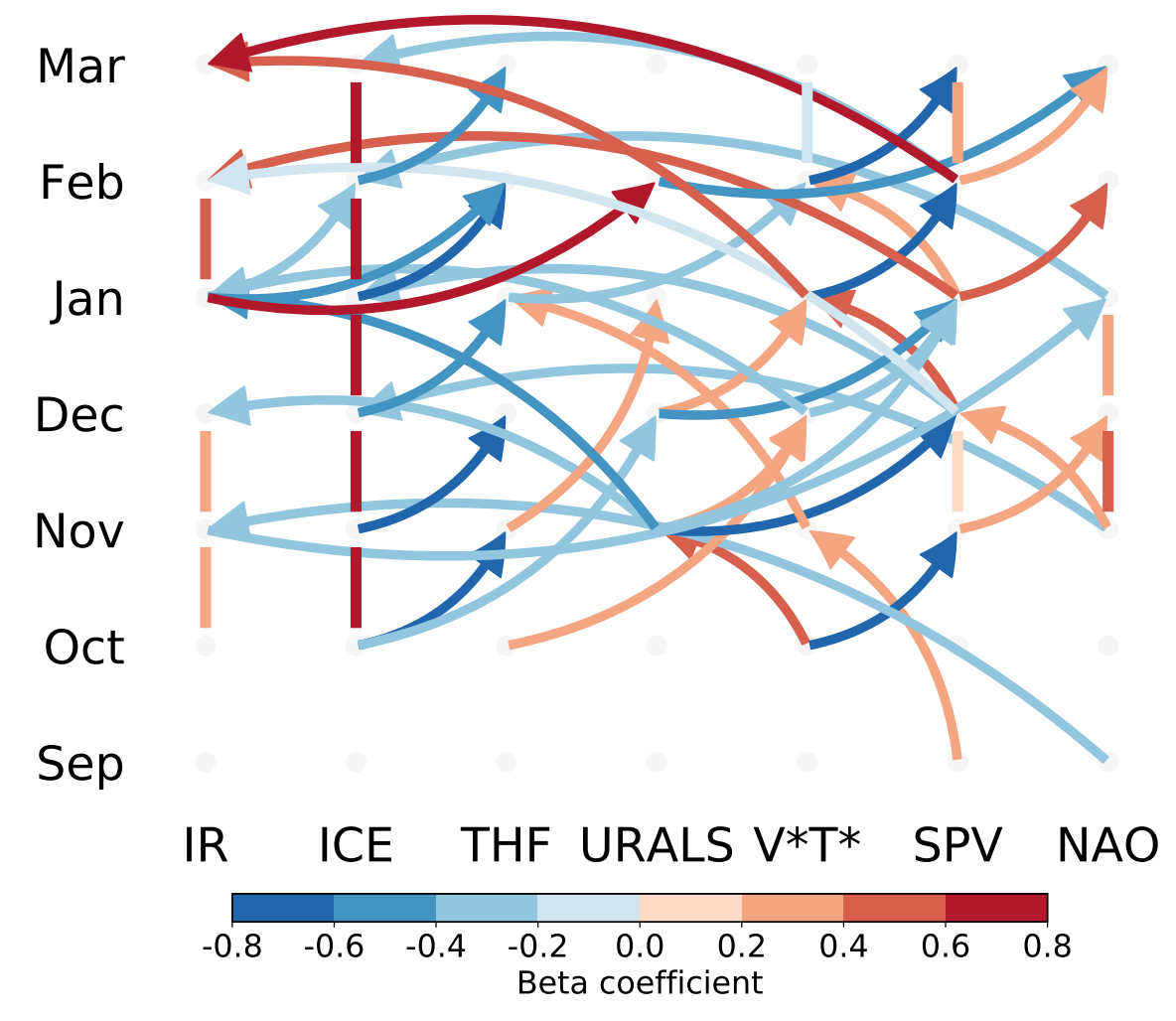

Figure S9: Monthly CEN as in Fig. S1a, but with the addition of downward longwave radiation (IR). 